\title{
Invariant Approximation Results via Common Fixed Point Theorems for Generalized Weak Contraction Maps
}

\author{
Savita Rathee, ${ }^{1}$ Anil Kumar, ${ }^{1}$ and Kenan Tas ${ }^{2}$ \\ ${ }^{1}$ Department of Mathematics, Maharshi Dayanand University, Rohtak, Haryana 124001, India \\ ${ }^{2}$ Department of Mathematics and Computer Science, Cankaya University, Ankara, Turkey
}

Correspondence should be addressed to Anil Kumar; anill_iit@yahoo.co.in

Received 25 March 2014; Revised 22 May 2014; Accepted 8 June 2014; Published 17 July 2014

Academic Editor: Erdal Karapinar

Copyright (C) 2014 Savita Rathee et al. This is an open access article distributed under the Creative Commons Attribution License, which permits unrestricted use, distribution, and reproduction in any medium, provided the original work is properly cited.

\begin{abstract}
A common fixed point theorem for generalized $(\varphi, \phi, L)_{f, g}$-weak contraction in a metric space is established. As an application, some common fixed point results in normed linear spaces are obtained. We also study some results on best approximation via common fixed point theorems. Our result improves some results from the existing literature. Some illustrative examples to highlight the realized improvements are also furnished.
\end{abstract}

\section{Introduction}

Let $(X, d)$ be a metric space. A mapping $T: X \rightarrow X$ is said to be contraction if there exists $0<k<1$ such that for all $x, y \in X$

$$
d(T x, T y) \leq k d(x, y) .
$$

If the metric space $(X, d)$ is complete, then the mapping satisfying (1) has a unique fixed point. This is known as the Banach contraction principle and is one of the significant results in nonlinear analysis. Inequality (1) also implies the continuity of $T$.

Due to its importance and usefulness, generalizations of the above contraction principle have been a very active field of research for the last four decades (see, e.g., [1-27]).

In 1997, Alber and Guerre-Delabriere [1] introduced the concept of weakly contractive mappings and proved the existence of fixed points for weakly contractive mappings in Hilbert spaces. Thereafter, Rhoades [2] assumed $\phi$-weakly contractive mappings $T: X \rightarrow X$ which satisfies the condition

$$
d(T x, T y) \leq d(x, y)-\phi(d(x, y))
$$

where $x, y \in X$ and $\phi:[0, \infty) \rightarrow[0, \infty)$ is a continuous and nondecreasing function such that $\phi(t)=0$ if and only if $t=0$ and shows that most of the results of Alber and Guerre-Delabriere [1] are still true in Banach spaces. If one takes $\phi(t)=k t$, where $0<k<1$, then (2) reduces to (1).

Recently, Zhang and Song [3] used the generalized $\phi$ weak contraction and proved the following result.

Theorem 1 (see [3]). Let $(X, d)$ be a complete metric space and let $T: X \rightarrow X$ be a map such that for all $x, y \in X$

$$
d(T x, T y) \leq M(x, y)-\phi(M(x, y)),
$$

where $\phi:[0, \infty) \rightarrow[0, \infty)$ is a lower semicontinuous function with $\phi(t)>0$ for $t \in(0, \infty)$ and $\phi(0)=0$,

$$
\begin{array}{r}
M(x, y)=\max \{d(x, y), d(T x, x), d(T y, y), \\
\left.\frac{1}{2}[d(y, T x)+d(x, T y)]\right\} .
\end{array}
$$

Then there exists the unique point $u \in X$ such that $u=T u$.

Further, using the control function defined by Khan et al. [4], the above result has been generalized by many authors (see [5-7]). On the other hand, Berinde [8] introduced the notion of $(k, L)$-weak contraction and proved that many wellknown contractive conditions do imply $(k, L)$-weak contraction. The concept of $(k, L)$-weak contraction does not ask $k+L$ 
to be less than 1 as happens in condition (1). Afterward, many authors study this new class of weak contraction and obtained some significant result (see [8-12]).

Definition 2. A map $T: X \rightarrow X$ is called generalized $(\varphi, \phi, L)_{f, g}$-weak contraction, if for each $x, y \in X$

$$
\varphi(d(T x, T y)) \leq \varphi(m(x, y))-\phi(m(x, y))+L \varphi(n(x, y)),
$$

where $f, g: X \rightarrow X, L \geq 0$,

$$
\begin{gathered}
m(x, y)=\max \{d(f x, g y), d(T x, f x), d(T y, g y), \\
\left.\frac{1}{2}[d(g y, T x)+d(f x, T y)]\right\}, \\
n(x, y)=\min \{[d(T x, f x)+d(T y, g y)], \\
d(g y, T x), d(f x, T y)\},
\end{gathered}
$$

and $\varphi:[0, \infty) \rightarrow[0, \infty)$ is a continuous monotone nondecreasing function with $\varphi(t)=0$ if and only if $t=0$ and $\phi:[0, \infty) \rightarrow[0, \infty)$ is a lower semicontinuous function from right such that $\phi$ is positive on $(0, \infty)$ and $\phi(0)=0$.

If $L=0$, then $T$ is said to be generalized $(\varphi, \phi)_{f, g}$-weak contraction. If $g=f$, then $T$ is called generalized $(\varphi, \phi)_{f^{-}}$ weak contraction. If $g=f=$ identity map, that is, $m(x, y)$ coincides with $M(x, y)$, then $T$ is called generalized $(\varphi, \phi)$ weak contraction which is exactly the maps studied by Doric [5]. Again, if $L=0$ and $\varphi(t)=t$, then $T$ is called generalized $\phi_{f, g}$-weak contraction which is the same as generalized $(f, g)$ weak contraction investigated by Akbar et al. (see [22]) and if $\phi(t)=(1-k) t$ for a constant $k$ with $0<k<1$, then $T$ is called generalized $(f, g)$-contraction which has been introduced by Song [21]. In Equation (5), if $f=g=I$ (identity map), then $T$ is called generalized $(\varphi, \phi, L)$-weak contraction.

Remark 3. It is obvious that the class of generalized $(\varphi, \phi, L)_{f, g}$-weak contraction contains the class of generalized $(\varphi, \phi)_{f, g}$-weak contraction and hence contains the class of generalized $\phi_{f, g}$-weak contraction, but the converse is not true, as shown by Remark 30.

In this paper, we prove that there is a unique common fixed point for generalized $(\varphi, \phi, L)_{f, g}$-weak contractive mappings in a metric space. As an application, some common fixed point results in normed linear space are obtained. We apply these theorems to obtain some results on invariant approximation. Our results generalize and extend the corresponding results of $[5,11,12,16-23,28]$ to the class of generalized $(\varphi, \phi, L)_{f, g}$-weak contractive maps.

\section{Preliminaries}

We need the following known definitions and standard notations in the sequel.

Let $M$ be a nonempty subset of a normed space $(X, \|$. $\|)$. The set $B_{M}(p)=\{x \in M:\|x-p\|=\operatorname{dist}(p, M)\}$ is called the set of best approximants to $p \in X$ out of $M$, where $\operatorname{dist}(p, M)=\inf \{\|y-p\|: y \in M\}$. We denote $\mathbb{N}$ and $\operatorname{cl}(M)$ (resp., $\operatorname{wcl}(M))$ by the set of positive integers and the closure (resp., weak closure) of a set $M$ in $X$. Let $f, T: M \rightarrow M$ be mappings. The set of fixed point of $T$ is denoted by $F(T)$. A point $x \in M$ is a coincidence point (common fixed point) of $f$ and $T$ if $f x=T x(f x=T x=x)$. The set of coincidence point of $f$ and $T$ is denoted by $C(f, T)$. The pair $\{f, T\}$ is called

(1) commuting if $f T x=T f x$ for all $x \in M$,

(2) compatible [13] if $\lim _{n \rightarrow \infty}\left\|T f x_{n}-f T x_{n}\right\|=0$ whenever $\left\{x_{n}\right\}$ is a sequence in $M$ such that $\lim _{n \rightarrow \infty} f x_{n}=$ $\lim _{n \rightarrow \infty} T x_{n}=t$ for some $t \in M$,

(3) weakly compatible [14] if $f T x=T f x$ for all $x \in$ $C(f, T)$,

(4) Banach operator pair [16] if the set $F(f)$ is $T$ invariant; that is, $T(F(f)) \subseteq F(f)$.

Apparently, a commuting pair $(f, T)$ is a Banach operator pair but not conversely. If $(f, T)$ is a Banach operator pair, then $(T, f)$ need not be Banach operator pair (see [16]).

The set $M$ is called $q$-star shaped with $q \in M$ if the segment $[q, x]=\{(1-k) q+k x: 0 \leq k \leq 1\}$ joining $q$ to $x$ is contained in $M$ for all $x \in M$. The map $f$ defined on $M$ is said to be (5) affine [18] if $M$ is convex and $f(k x+(1-k) y)=$ $k f x+(1-k) f y$ for all $x, y \in M$ and $k \in[0,1]$ and (6) $q$-affine [18] if $M$ is $q$-star shaped and $f(k x+(1-k) q)=k f x+(1-k) q$ for all $x \in M$ and $k \in[0,1]$.

Suppose that $M$ is $q$-star shaped with $q \in F(f)$ and is both $T$ - and $f$-invariant. Then $T$ and $f$ are called

(1) $C_{q}$-commuting [18] if $f T x=T f x$ for all $x \in C_{q}(f, T)$, where $C_{q}(f, T)=\cup\left\{C\left(f, T_{k}\right): 0 \leq k \leq 1\right\}$, where $T_{k}(x)=(1-k) q+k T x$,

(2) $R$-subweakly commuting on $M$ [15] if, for all $x \in M$, there exists a real number $R>0$ such that $\| T f x-$ $f T x \| \leq R \operatorname{dist}(f x,[q, T x])$.

Definition 4. A map $T: M \rightarrow X$ is said to be demiclosed at $0 \in X$ if whenever $\left\{x_{n}\right\}$ is a sequence in $M$ such that $\left\{x_{n}\right\}$ converges weakly to $x \in M$ and $\left\{T x_{n}\right\}$ converges strongly to 0 ; then $T x=0$.

Definition 5. A Banach space $X$ is said to satisfy Opial's condition if whenever $\left\{x_{n}\right\}$ is a sequence in $X$ such that $\left\{x_{n}\right\}$ converges weakly to $x \in X$; the inequality

$$
\lim \inf _{n \rightarrow \infty}\left\|x_{n}-x\right\|<\lim \inf _{n \rightarrow \infty}\left\|x_{n}-y\right\|
$$

holds for all $y \neq x$. Every Hilbert space and the space $l_{p}(1<$ $p<\infty)$ satisfy Opial's condition.

Lemma 6. If $\lim _{n \rightarrow \infty} d\left(y_{n+1}, y_{n}\right)=0$ and $\left\{y_{2 n}\right\}$ is a Cauchy sequence, then $\left\{y_{n}\right\}$ is also a Cauchy sequence.

Proof. Since $\left\{y_{2 n}\right\}$ is a Cauchy sequence, we have that, for a given $\varepsilon>0$, there is a $n_{0}>0$ such that for every $n, m>n_{0}$ we have $d\left(y_{2 n}, y_{2 m}\right) \leq \varepsilon / 3$. Also, since $\lim _{n \rightarrow \infty} d\left(y_{n+1}, y_{n}\right)=0$ we have that, given $\varepsilon>0$, there is a $n_{1}>0$ such that, for every $n, m>n_{1}, d\left(y_{n+1}, y_{n}\right)<\varepsilon / 3$. Now, suppose $m, n>m_{0}=$ $\max \left\{n_{0}, n_{1}\right\}$; then we have three possibilities. 
(a) If $m$ and $n$ are both even numbers, let $m=2 k, n=2 s$; then $d\left(y_{m}, y_{n}\right)=d\left(y_{2 k}, y_{2 s}\right) \leq \varepsilon / 3<\varepsilon$.

(b) If $m$ is even and $n$ is odd, let $m=2 k, n=2 s+1$; then by the triangle inequality $d\left(y_{m}, y_{n}\right) \leq d\left(y_{m}, y_{n+1}\right)+$ $d\left(y_{n+1}, y_{n}\right)=d\left(y_{2 k}, y_{2 s+2}\right)+d\left(y_{2 s+2}, y_{2 s+1}\right) \leq \varepsilon / 3+$ $\varepsilon / 3=2 \varepsilon / 3<\varepsilon$.

(c) If $m, n$ are both odd numbers, let $m=2 k+1, n=$ $2 s+1$; then using the previous estimate $d\left(y_{m}, y_{n}\right)=$ $d\left(y_{2 k+1}, y_{2 s+1}\right) \leq d\left(y_{2 k+1}, y_{2 s+2}\right)+d\left(y_{2 s+2}, y_{2 s+1}\right) \leq$ $2 \varepsilon / 3+\varepsilon / 3=\varepsilon$.

Therefore $\left\{y_{n}\right\}$ is Cauchy.

\section{Main Results}

The following result extends and improves Theorem 2.2 of [5], Theorem 2.4 of [11], Theorem 2.1 of [12], and Theorem 2.1 of [18].

Theorem 7. Let $M$ be a nonempty subset of a metric space $(X, d)$ and $f, g$, and $T$ are self-mappings of $M$ such that $c l(T(M)) \subseteq f(M) \cap g(M)$. Assume that cl $(T(M))$ is complete and $T$ is generalized $(\varphi, \phi, L)_{f, g}$-weak contraction. Then the pairs $(T, f)$ and $(T, g)$ have a unique point of coincidence in M. Also, if the pairs $(T, f)$ and $(T, g)$ are weakly compatible, then $M \cap F(T) \cap F(f) \cap F(g)$ is singleton.

Proof. Let $x_{0} \in M$ be an arbitrary point. Since $\operatorname{cl}(T(M)) \subseteq$ $f(M) \cap g(M)$, we can choose a point $x_{1} \in M$ such that $y_{0}=$ $T x_{0}=f x_{1}$. Similarly a point $x_{2} \in M$ can be chosen such that $y_{1}=T x_{1}=g x_{2}$. Continuing this process, we obtain a sequence $\left\{y_{n}\right\}$ in $M$ such that $y_{2 n}=T x_{2 n}=f x_{2 n+1}$ and $y_{2 n+1}=T x_{2 n+1}=g x_{2 n+2}$ for every $n \geq 0$. If, for some $n$, $y_{2 n+1}=y_{2 n}$, then $\left\{y_{n}\right\}$ turns out to be a constant sequence and hence it is Cauchy. Now suppose that $y_{2 n+1} \neq y_{2 n}$.

Using the fact that $T$ is generalized $(\varphi, \phi, L)_{f, g}$-weak contraction, for each $n \geq 0$, we have

$$
\begin{aligned}
\varphi\left(d\left(y_{2 n+1}, y_{2 n}\right)\right)= & \varphi\left(d\left(T x_{2 n+1}, T x_{2 n}\right)\right) \\
\leq & \varphi\left(m\left(x_{2 n+1}, x_{2 n}\right)\right)-\phi\left(m\left(x_{2 n+1}, x_{2 n}\right)\right) \\
& +L \varphi\left(n\left(x_{2 n+1}, x_{2 n}\right)\right)
\end{aligned}
$$

where

$$
\begin{aligned}
& m\left(x_{2 n+1}, x_{2 n}\right) \\
& =\max \left\{d\left(f x_{2 n+1}, g x_{2 n}\right), d\left(T x_{2 n+1}, f x_{2 n+1}\right), d\left(T x_{2 n}, g x_{2 n}\right)\right. \\
& \left.\quad \frac{1}{2}\left[d\left(g x_{2 n}, T x_{2 n+1}\right)+d\left(f x_{2 n+1}, T x_{2 n}\right)\right]\right\} \\
& n\left(x_{2 n+1}, x_{2 n}\right) \\
& =\min \left\{\left[d\left(T x_{2 n+1}, f x_{2 n+1}\right)+d\left(T x_{2 n}, g x_{2 n}\right)\right]\right. \\
& \left.\quad d\left(g x_{2 n}, T x_{2 n+1}\right), d\left(f x_{2 n+1}, T x_{2 n}\right)\right\}
\end{aligned}
$$

$$
\begin{gathered}
=\min \left\{\left[d\left(y_{2 n+1}, y_{2 n}\right)+d\left(y_{2 n}, y_{2 n-1}\right)\right],\right. \\
\left.d\left(y_{2 n-1}, y_{2 n+1}\right), 0\right\}=0 .
\end{gathered}
$$

Since $\varphi(0)=0$, therefore we have

$$
\varphi\left(d\left(y_{2 n+1}, y_{2 n}\right)\right) \leq \varphi\left(m\left(x_{2 n+1}, x_{2 n}\right)\right)-\phi\left(m\left(x_{2 n+1}, x_{2 n}\right)\right) \text {. }
$$

This implies

$$
\varphi\left(d\left(y_{2 n+1}, y_{2 n}\right)\right) \leq \varphi\left(m\left(x_{2 n+1}, x_{2 n}\right)\right) .
$$

As $\varphi$ is a nondecreasing function, therefore for each $n \geq 0$ we have

$$
d\left(y_{2 n+1}, y_{2 n}\right) \leq m\left(x_{2 n+1}, x_{2 n}\right)
$$

Now from the triangle inequality for $d$ we have

$$
\begin{aligned}
& m\left(x_{2 n+1}, x_{2 n}\right) \\
&=\max \left\{d\left(f x_{2 n+1}, g x_{2 n}\right), d\left(T x_{2 n+1}, f x_{2 n+1}\right), d\left(T x_{2 n}, g x_{2 n}\right),\right. \\
&\left.\frac{1}{2}\left[d\left(g x_{2 n}, T x_{2 n+1}\right)+d\left(f x_{2 n+1}, T x_{2 n}\right)\right]\right\} \\
&=\max \left\{d\left(y_{2 n}, y_{2 n-1}\right), d\left(y_{2 n+1}, y_{2 n}\right), d\left(y_{2 n}, y_{2 n-1}\right),\right. \\
&\left.\frac{1}{2}\left[d\left(y_{2 n-1}, y_{2 n+1}\right)+d\left(y_{2 n}, y_{2 n}\right)\right]\right\} \\
& \leq \max \left\{d\left(y_{2 n}, y_{2 n-1}\right), d\left(y_{2 n+1}, y_{2 n}\right),\right. \\
& \\
&\left.\frac{1}{2}\left[d\left(y_{2 n-1}, y_{2 n}\right)+d\left(y_{2 n}, y_{2 n+1}\right)\right]\right\} .
\end{aligned}
$$

If $d\left(y_{2 n+1}, y_{2 n}\right)>d\left(y_{2 n}, y_{2 n-1}\right)$, then $m\left(x_{2 n+1}, x_{2 n}\right)=$ $d\left(y_{2 n+1}, y_{2 n}\right)>0$. It furthermore implies that

$$
\varphi\left(d\left(y_{2 n+1}, y_{2 n}\right)\right) \leq \varphi\left(d\left(y_{2 n+1}, y_{2 n}\right)\right)-\phi\left(d\left(y_{2 n+1}, y_{2 n}\right)\right) .
$$

This is a contradiction; therefore we have

$$
d\left(y_{2 n+1}, y_{2 n}\right) \leq m\left(x_{2 n+1}, x_{2 n}\right) \leq d\left(y_{2 n}, y_{2 n-1}\right) .
$$

Similarly, it can be shown that

$$
d\left(y_{2 n}, y_{2 n-1}\right) \leq m\left(x_{2 n}, x_{2 n-1}\right) \leq d\left(y_{2 n-1}, y_{2 n-2}\right) \text {. }
$$

Therefore, for each $n \geq 0$, we have

$$
d\left(y_{n+1}, y_{n}\right) \leq m\left(x_{n+1}, x_{n}\right) \leq d\left(y_{n}, y_{n-1}\right) .
$$

Thus, the sequence $\left\{d\left(y_{n+1}, y_{n}\right)\right\}$ is monotone nonincreasing and bounded. So there exist $r \geq 0$ such that

$$
\lim _{n \rightarrow \infty} d\left(y_{n+1}, y_{n}\right)=\lim _{n \rightarrow \infty} m\left(x_{n+1}, x_{n}\right)=r .
$$


After letting $n \rightarrow \infty$ in (10), we obtain $\varphi(r) \leq \varphi(r)-\phi(r)$, which is a contradiction unless $r=0$.

Hence

$$
\lim _{n \rightarrow \infty} d\left(y_{n+1}, y_{n}\right)=0 \text {. }
$$

Because of (19) and Lemma 6, to show $\left\{y_{n}\right\}$ to be a Cauchy sequence in $M$, it is sufficient to show that $\left\{y_{2 n}\right\}$ is a Cauchy in $M$.

Suppose not, then there exists $\epsilon>0$ for which we can find subsequences $\left\{y_{2 m(k)}\right\}$ and $\left\{y_{2 n(k)}\right\}$ of $\left\{y_{2 n}\right\}$ with $n(k)>$ $m(k)>k$ such that, for every $k, d\left(y_{2 m(k)}, y_{2 n(k)}\right) \geq \epsilon$ and $d\left(y_{2 m(k)}, y_{2 n(k)-2}\right)<\epsilon$. So we have

$$
\begin{aligned}
\epsilon \leq & d\left(y_{2 m(k)}, y_{2 n(k)}\right) \\
\leq & d\left(y_{2 m(k)}, y_{2 n(k)-2}\right)+d\left(y_{2 n(k)-2}, y_{2 n(k)-1}\right) \\
& +d\left(y_{2 n(k)-1}, y_{2 n(k)}\right) \\
& <\epsilon+d\left(y_{2 n(k)-2}, y_{2 n(k)-1}\right)+d\left(y_{2 n(k)-1}, y_{2 n(k)}\right) .
\end{aligned}
$$

Now using (19), we have

$$
\lim _{k \rightarrow \infty} d\left(y_{2 m(k)}, y_{2 n(k)}\right)=\epsilon
$$

Moreover, using the known relation $|d(x, z)-d(x, y)| \leq$ $d(y, z)$, we obtain

$$
\left|d\left(y_{2 m(k)}, y_{2 n(k)-1}\right)-d\left(y_{2 m(k)}, y_{2 n(k)}\right)\right| \leq d\left(y_{2 n(k)}, y_{2 n(k)-1}\right) .
$$

Then by using (19) and (21), we get

$$
\lim _{k \rightarrow \infty} d\left(y_{2 m(k)}, y_{2 n(k)-1}\right)=\epsilon .
$$

Again from the relation

$$
\begin{aligned}
& \left|d\left(y_{2 n(k)-1}, y_{2 m(k)+1}\right)-d\left(y_{2 n(k)-1}, y_{2 m(k)}\right)\right| \\
& \quad \leq d\left(y_{2 m(k)+1}, y_{2 m(k)}\right),
\end{aligned}
$$

using (19) and (23), we get

$$
\lim _{k \rightarrow \infty} d\left(y_{2 n(k)-1}, y_{2 m(k)+1}\right)=\epsilon .
$$

Now from

$$
\begin{aligned}
& \left|d\left(y_{2 m(k)+1}, y_{2 n(k)}\right)-d\left(y_{2 m(k)+1}, y_{2 n(k)-1}\right)\right| \\
& \quad \leq d\left(y_{2 n(k)}, y_{2 n(k)-1}\right),
\end{aligned}
$$

using (19) and (25), we get

$$
\lim _{k \rightarrow \infty} d\left(y_{2 m(k)+1}, y_{2 n(k)}\right)=\epsilon .
$$

Again, using the fact that $T$ is generalized $(\varphi, \phi, L)_{f, g}$-weak contraction, we get

$$
\begin{aligned}
\varphi( & \left.\left(y_{2 m(k)+1}, y_{2 n(k)}\right)\right) \\
= & \varphi\left(d\left(T x_{2 m(k)+1}, T x_{2 n(k)}\right)\right) \\
\leq & \varphi\left(m\left(x_{2 m(k)+1}, x_{2 n(k)}\right)\right)-\phi\left(m\left(x_{2 m(k)+1}, x_{2 n(k)}\right)\right) \\
& \quad+L \varphi\left(n\left(x_{2 m(k)+1}, x_{2 n(k)}\right)\right),
\end{aligned}
$$

where

$$
\begin{aligned}
& m\left(x_{2 m(k)+1}, x_{2 n(k)}\right) \\
&=\max \left\{d\left(f x_{2 m(k)+1}, g x_{2 n(k)}\right), d\left(T x_{2 m(k)+1}, f x_{2 m(k)+1}\right),\right. \\
& d\left(T x_{2 n(k)}, g x_{2 n(k)}\right), \\
&\left.\frac{1}{2}\left[d\left(g x_{2 n(k)}, T x_{2 m(k)+1}\right)+d\left(f x_{2 m(k)+1}, T x_{2 n(k)}\right)\right]\right\} \\
&=\max \left\{d\left(y_{2 m(k)}, y_{2 n(k)-1}\right), d\left(y_{2 m(k)+1}, y_{2 m(k)}\right),\right. \\
& d\left(y_{2 n(k)}, y_{2 n(k)-1}\right), \\
&\left.\frac{1}{2}\left[d\left(y_{2 n(k)-1}, y_{2 m(k)+1}\right)+d\left(y_{2 m(k)}, y_{2 n(k)}\right)\right]\right\} \\
& \longrightarrow \max \left\{\epsilon, 0,0, \frac{1}{2}(\epsilon+\epsilon)\right\}=\epsilon \quad \text { as } k \longrightarrow \infty .
\end{aligned}
$$$$
\text { [Using (19), (21), (23), (25)] }
$$

And

$$
\begin{aligned}
& n\left(x_{2 m(k)+1}, x_{2 n(k)}\right) \\
& =\min \left\{\left[d\left(T x_{2 m(k)+1}, f x_{2 m(k)+1}\right)+d\left(T x_{2 n(k)}, g x_{2 n(k)}\right)\right],\right. \\
& \left.d\left(g x_{2 n(k)}, T x_{2 m(k)+1}\right), d\left(f x_{2 m(k)+1}, T x_{2 n(k)}\right)\right\} \\
& =\min \left\{\left[d\left(y_{2 m(k)+1}, y_{2 m(k)}\right)+d\left(y_{2 n(k)}, y_{2 n(k)-1}\right)\right]\right. \text {, } \\
& \left.d\left(y_{2 n(k)-1}, y_{2 m(k)+1}\right), d\left(y_{2 m(k)}, y_{2 n(k)}\right)\right\} \\
& \longrightarrow 0 \quad \text { as } k \longrightarrow \infty \text {. [Using (19), (21), (25)] }
\end{aligned}
$$

Letting $k \rightarrow \infty$ in (28), we get

$$
\varphi(\epsilon) \leq \varphi(\epsilon)-\phi(\epsilon),
$$

which is a contradiction with $\epsilon>0$. Thus $\left\{y_{2 n}\right\}$ is a Cauchy sequence and hence $\left\{y_{n}\right\}$ is a Cauchy sequence; therefore by the completeness of $\operatorname{cl}(T(M))$ there is some $z \in \operatorname{cl}(T(M))$ such that

$$
\lim _{n \rightarrow \infty} y_{n}=\lim _{n \rightarrow \infty} T x_{n}=\lim _{n \rightarrow \infty} f x_{2 n+1}=z=\lim _{n \rightarrow \infty} g x_{2 n+2} .
$$

Further, $\operatorname{cl}(T(M)) \subseteq f(M) \cap g(M)$; therefore there exist $u, v \in$ $M$ such that

$$
f u=z=g v .
$$

Since $T$ is generalized $(\varphi, \phi, L)_{f, g}$-weak contraction, therefore

$$
\begin{aligned}
& \varphi\left(d\left(T u, f x_{2 n+1}\right)\right) \\
& \quad=\varphi\left(d\left(T u, T x_{2 n}\right)\right) \\
& \quad \leq \varphi\left(m\left(u, x_{2 n}\right)\right)-\phi\left(m\left(u, x_{2 n}\right)\right)+L \varphi\left(n\left(u, x_{2 n}\right)\right),
\end{aligned}
$$


where

$$
\begin{aligned}
& m\left(u, x_{2 n}\right) \\
& =\max \left\{d\left(f u, g x_{2 n}\right), d(T u, f u), d\left(T x_{2 n}, g x_{2 n}\right),\right. \\
& \left.\quad \frac{1}{2}\left[d\left(g x_{2 n}, T u\right)+d\left(f u, T x_{2 n}\right)\right]\right\} \\
& n\left(u, x_{2 n}\right) \\
& =\min \left\{\left[d(T u, f u)+d\left(T x_{2 n}, g x_{2 n}\right)\right]\right. \\
& \left.\quad d\left(g x_{2 n}, T u\right), d\left(f u, T x_{2 n}\right)\right\} .
\end{aligned}
$$

Now using (32) and (33), we can write

$$
m\left(u, x_{2 n}\right) \longrightarrow d(T u, z), \quad n\left(u, x_{2 n}\right) \longrightarrow 0 \quad \text { as } n \longrightarrow \infty \text {. }
$$

Therefore, letting $n \rightarrow \infty$ in (34), we get

$$
\varphi(d(T u, z)) \leq \varphi(d(T u, z))-\phi(d(T u, z)) .
$$

This is true only if $d(T u, z)=0$; that is, $T u=z=f u$ and $u$ is coincidence point of $T$ and $f$.

Since $T$ is generalized $(\varphi, \phi, L)_{f, g}$-weak contraction, therefore

$$
\begin{aligned}
\varphi( & \left.d\left(g x_{2 n+2}, T v\right)\right) \\
& =\varphi\left(d\left(T x_{2 n+1}, T v\right)\right) \\
& \leq \varphi\left(m\left(x_{2 n+1}, v\right)\right)-\phi\left(m\left(x_{2 n+1}, v\right)\right)+L \varphi\left(n\left(x_{2 n+1}, v\right)\right),
\end{aligned}
$$

where

$$
\begin{aligned}
& m\left(x_{2 n+1}, v\right) \\
& =\max \left\{d\left(f x_{2 n+1}, g v\right), d\left(T x_{2 n+1}, f x_{2 n+1}\right), d(T v, g v),\right. \\
& \left.\quad \frac{1}{2}\left[d\left(g v, T x_{2 n+1}\right)+d\left(f x_{2 n+1}, T v\right)\right]\right\}, \\
& n\left(x_{2 n+1}, v\right) \\
& =\min \left\{\left[d\left(T x_{2 n+1}, f x_{2 n+1}\right)+d(T v, g v)\right],\right. \\
& \left.\quad d\left(g v, T x_{2 n+1}\right), d\left(f x_{2 n+1}, T v\right)\right\} .
\end{aligned}
$$

Therefore, (32) and (33) imply

$$
\begin{array}{r}
m\left(x_{2 n+1}, v\right) \longrightarrow d(T v, z), \quad n\left(x_{2 n+1}, v\right) \\
\text { as } n \longrightarrow 0 \\
\longrightarrow \infty .
\end{array}
$$

Then, letting $n \rightarrow \infty$ in (38), we obtain

$$
\varphi(d(z, T v)) \leq \varphi(d(z, T v))-\phi(d(z, T v)) .
$$

This is true only if $d(T v, z)=0$; that is, $T v=z=g v$ and $v$ is coincidence point of $T$ and $g$. Thus the pairs $(T, f)$ and $(T, g)$ have a common point of coincidence in $M$.

If the pairs $(T, f)$ and $(T, g)$ are weakly compatible, then $T z=T f u=f T u=f z$ and $T z=T g v=g T v=g z$ and hence $T z=f z=g z$. Now, we have to show that $T z=z$.
Further, using the fact that $T$ is generalized $(\varphi, \phi, L)_{f, g^{-}}$ weak contraction, we have

$$
\begin{aligned}
\varphi(d(T z, z)) & =\varphi(d(T z, T v)) \\
& \leq \varphi(m(z, v))-\phi(m(z, v))+L \varphi(n(z, v)),
\end{aligned}
$$

where

$$
\begin{aligned}
& m(z, v) \\
& =\max \{d(f z, g v), d(T z, f z), d(T v, g v), \\
& \left.\frac{1}{2}[d(g v, T z)+d(f z, T v)]\right\} \\
& =\max \left\{d(T z, z), 0,0, \frac{1}{2}[d(z, T z)+d(T z, z)]\right\} \\
& =d(T z, z), \\
& n(z, v) \\
& =\min \{[d(T z, f z)+d(T v, g v)], \\
& d(g v, T z), d(f z, T v)\}=0 .
\end{aligned}
$$

Then from (42), we get

$$
\varphi(d(T z, z)) \leq \varphi(d(T z, z))-\phi(d(T z, z)) .
$$

This is true only if $T z=z$ which implies $T z=f z=g z=z$.

Moreover, it can be easily shown that this $z$ is unique and hence $M \cap F(T) \cap F(f) \cap F(g)$ is singleton.

If we take $f=g=$ identity mapping in Theorem 7 , then we have the following result.

Corollary 8. Let $M$ be a nonempty subset of a metric space $(X, d)$ and let $T$ be a self-mapping of $M$ such that $c l(T(M)) \subseteq$ $M$. Assume that cl $(T(M))$ is complete and $T$ is generalized $(\varphi, \phi, L)$-weak contraction. Then $M \cap F(T)$ is singleton.

Corollary 9 (see [5], Theorem 2.2). Let $(X, d)$ be a complete metric space and let $T$ be a self-mapping of $X$. If $T$ is generalized $(\varphi, \phi)$-weak contractions, then there is a unique fixed point of $T$.

In Corollary 8 , if $\varphi(t)=t$ and $L=0$, then Theorem 1 can be obtained as a particular case of the following result.

Corollary 10. Let $M$ be a subset of a metric space $(X, d)$ and let $T$ be a self-mapping of $M$ such that $c l(T(M)) \subseteq M$. Assume that $\mathrm{cl}(T(M))$ is complete and $T$ is generalized $\phi$-weak contractions. Then $M \cap F(T)$ is singleton.

Remark 11. (1) In Theorem 7, if $L=0, \varphi(t)=t$ and $\phi(t)=$ $(1-k) t$ for a constant $k$ with $0<k<1$, then we obtain Theorem 2.1 of Song [21] as a particular case of Theorem 7.

(2) In Theorem 7, if $L=0, \varphi(t)=t$ and $\phi(t)=(1-k) t$ for a constant $k$ with $0<k<1$, then for $g=f=$ identity map (resp., $g=f$ ) we obtain Theorem 2.4 of Berinde [11] (resp., Theorem 2.1 of Abbas and Ilić [12]) as a particular case of Theorem 7. 
As an application of Corollary 8, we obtain the following general common fixed point result.

Theorem 12. Let $M$ be a nonempty subset of a metric space $(X, d)$ and $T, f$, and $g$ are self-maps of $M$. Assume that $\mathrm{cl}(T(M))$ is complete, $T$ is generalized $(\varphi, \phi, L)_{f, g^{-w e a k}}$ contraction, $F(f) \cap F(g)$ is nonempty, and $c l(T(F(f) \cap F(g))) \subseteq$ $F(f) \cap F(g)$. Then $M \cap F(T) \cap F(f) \cap F(g)$ is singleton.

Proof. $\mathrm{cl}(T(F(f) \cap F(g)))$ is complete by the completeness of $\operatorname{cl}(T(M))$. Also, for all $x, y \in F(f) \cap F(g)$, we have by generalized $(\varphi, \phi, L)_{f, g}$-weak contractiveness of $T$ :

$$
\begin{aligned}
\varphi( & (T x, T y)) \\
& \leq \varphi(m(x, y))-\phi(m(x, y))+L \varphi(n(x, y)) \\
& =\varphi(M(x, y))-\phi(M(x, y))+L \varphi(N(x, y)),
\end{aligned}
$$

where $N(x, y)=\min \{[d(T x, x)+d(T y, y)], d(y, T x), d(x$, $T y)\}$.

Hence $T$ is generalized $(\varphi, \phi, L)$-weak contraction mapping on $F(f) \cap F(g)$. Ascl $(T(F(f) \cap F(g))) \subseteq F(f) \cap F(g)$, thus, by Corollary $8, T$ has a unique fixed point $z$ in $F(f) \cap F(g)$ and, consequently, $M \cap F(T) \cap F(f) \cap F(g)$ is singleton.

Corollary 13. Let $M$ be a nonempty subset of a metric space $(X, d)$ and $(T, f)$ and $(T, g)$ are Banach operator pairs on $M$. Assume that cl $(T(M))$ is complete, $T$ is generalized $(\varphi, \phi, L)_{f, g^{-}}$ weak contraction, and $F(f) \cap F(g)$ is nonempty and closed. Then $M \cap F(T) \cap F(f) \cap F(g)$ is singleton.

Proof. Since $(T, f)$ and $(T, g)$ are Banach operator pairs on $M$, therefore $T(F(f) \cap F(g)) \subseteq F(f) \cap F(g)$; then by closedness of $F(f) \cap F(g)$ we have $\operatorname{cl}(T(F(f) \cap F(g))) \subseteq F(f) \cap F(g)$. By Theorem 12, T has a unique fixed point $z$ in $F(f) \cap F(g)$ and, consequently, $M \cap F(T) \cap F(f) \cap F(g)$ is singleton.

In Theorem 12, if $L=0$, then we easily obtain the following results which properly contain Theorem 3.3 of Akbar et al. [22].

Corollary 14. Let $M$ be a nonempty subset of a metric space $(X, d)$ and $T, f$, and $g$ are self-maps of $M$. Assume that $\mathrm{cl}(T(M))$ is complete, $T$ is generalized $(\varphi, \phi)_{f, g}$-weak contraction, $F(f) \cap F(g)$ is nonempty, and $c l(T(F(f) \cap F(g))) \subseteq$ $F(f) \cap F(g)$. Then $M \cap F(T) \cap F(f) \cap F(g)$ is singleton.

In Corollary 14, if $\varphi(t)=t$ and $\phi(t)=(1-k) t$ for a constant $k$ with $0<k<1$, then for $g=f$ we obtain the following result which improves Lemma 3.1 of Chen and $\mathrm{Li}$ [16] and Theorem 2.2 of Al-Thagafi and Shahzad [19].

Corollary 15. Let $M$ be a nonempty subset of a metric space $(X, d)$ and $T, f$ and $g$ are self-maps of $M$. Assume that $c l(T(M))$ is complete, $T$ is generalized $f$-contraction, $F(f) \cap$ $F(g)$ is nonempty, and $c l(T(F(f) \cap F(g))) \subseteq F(f) \cap F(g)$. Then $M \cap F(T) \cap F(f) \cap F(g)$ is singleton.

The following theorem properly contains Theorem 3.8 and Corollary 3.9 of Akbar et al. [22].
Theorem 16. Let $M$ be a nonempty subset of a normed [resp., Banach] space $X$ and $T, f$, and $g$ are self-maps of $M$. Suppose that $F(f) \cap F(g)$ is q-star shaped, $c l(T(F(f) \cap F(g))) \subseteq F(f) \cap$ $F(g)[$ resp., $w c l(T(F(f) \cap F(g))) \subseteq F(f) \cap F(g)]$, and $T, f$, and $g$ satisfy

$$
\begin{aligned}
& \varphi(k\|T x-T y\|) \\
& \quad \leq \varphi\left(m_{1}(x, y)\right)-k \phi\left(m_{1}(x, y)\right)+k L \varphi\left(n_{1}(x, y)\right),
\end{aligned}
$$

for all $k \in(0,1)$ and $x, y \in M$, where

(a) $\varphi:[0, \infty) \rightarrow[0, \infty)$ is a continuous monotone nondecreasing function with $\varphi(t)=0$ if and only if $t=0$,

(b) $\phi:[0, \infty) \rightarrow[0, \infty)$ is a lower semicontinuous function from right such that $\phi$ is positive on $(0, \infty)$ and $\phi(0)=0$,

(c) $m_{1}(x, y)=\max \{\|f x-g y\|, \operatorname{dist}(f x,[q, T x]), \operatorname{dist}(g y$, $[q, T y]),(1 / 2)[\operatorname{dist}(g y,[q, T x])+\operatorname{dist}(f x,[q, T y])]\}$,

(d) $n_{1}(x, y)=\min \{[\operatorname{dist}(f x,[q, T x])+\operatorname{dist}(g y,[q, T y])]$, $\operatorname{dist}(g y,[q, T x]), \operatorname{dist}(f x,[q, T y])\}$.

Then $M \cap F(T) \cap F(f) \cap F(g) \neq \phi$ provided that $c l(T(M))$ is compact [resp., wcl $(T(M))$ is weakly compact] and Tis continuous [resp., I - T is demiclosed at 0 , where I stands for identity map].

Proof. For each $n \in \mathbb{N}$, we define $T_{n}: F(f) \cap F(g) \rightarrow F(f) \cap$ $F(g)$ by $T_{n} x=\left(1-k_{n}\right) q+k_{n} T x$ for all $x \in F(f) \cap F(g)$ and a fixed sequence of real numbers $k_{n}\left(0<k_{n}<1\right)$ converging to 1. Since $F(f) \cap F(g)$ is $q$-star shaped and $\operatorname{cl}(T(F(f) \cap F(g))) \subseteq$ $F(f) \cap F(g)[$ resp. $\operatorname{wcl}(T(F(f) \cap F(g))) \subseteq F(f) \cap F(g)]$, we have $\operatorname{cl}\left(T_{n}(F(f) \cap F(g))\right) \subseteq F(f) \cap F(g)\left[\right.$ resp., $w c l\left(T_{n}(F(f) \cap\right.$ $F(g))) \subseteq F(f) \cap F(g)]$ for each $n \in \mathbb{N}$. Let $\phi_{n}=k_{n} \phi$ and $L_{n}=k_{n} L$. Then by using (46), for all $x, y \in F(f) \cap F(g)$, we have

$$
\begin{aligned}
\varphi( & \left.\left\|T_{n} x-T_{n} y\right\|\right) \\
& =\varphi\left(k_{n}\|T x-T y\|\right) \\
& \leq \varphi\left(m_{1}(x, y)\right)-k_{n} \phi\left(m_{1}(x, y)\right)+k_{n} L \varphi\left(n_{1}(x, y)\right) \\
& =\varphi\left(m_{1}(x, y)\right)-\phi_{n}\left(m_{1}(x, y)\right)+L_{n} \varphi\left(n_{1}(x, y)\right),
\end{aligned}
$$

where

$$
\begin{aligned}
m_{1}(x, y) & \\
=\max & \{\|f x-g y\|, \operatorname{dist}(f x,[q, T x]), \operatorname{dist}(g y,[q, T y]), \\
& \left.\frac{1}{2}[\operatorname{dist}(g y,[q, T x])+\operatorname{dist}(f x,[q, T y])]\right\} \\
\leq \max & \left\{\|f x-g y\|,\left\|f x-T_{n} x\right\|,\left\|g y-T_{n} y\right\|,\right. \\
& \left.\frac{1}{2}\left[\left\|g y-T_{n} x\right\|+\left\|f x-T_{n} y\right\|\right]\right\},
\end{aligned}
$$




$$
\begin{aligned}
n_{1}(x, y) & \\
=\min \{ & {[\operatorname{dist}(f x,[q, T x])+\operatorname{dist}(g y,[q, T y])], } \\
& \operatorname{dist}(g y,[q, T x]), \operatorname{dist}(f x,[q, T y])\} \\
\leq \min \{[ & {\left[f x-T_{n} x\|+\| g y-T_{n} y \|\right], } \\
& \left.\left\|g y-T_{n} x\right\|,\left\|f x-T_{n} y\right\|\right\} .
\end{aligned}
$$

Clearly, $\phi_{n}:[0, \infty) \rightarrow[0, \infty)$ is a lower semicontinuous function from right such that $\phi_{n}$ is positive on $(0, \infty)$ and $\phi_{n}(0)=0$ and $L_{n} \geq 0$. Thus, for each $n \in \mathbb{N}, T_{n}$ is generalized $\left(\varphi, \phi_{n}, L_{n}\right)_{f, g}$-weak contraction. As $\operatorname{cl}(T(M))$ is compact [resp., $\operatorname{wcl}(T(M))$ is weakly compact], therefore, for each $n \in \mathbb{N}, \operatorname{cl}\left(T_{n}(M)\right)$ is compact [resp., $\operatorname{wcl}\left(T_{n}(M)\right)$ is weakly compact]. Thus, $\operatorname{cl}\left(T_{n}(M)\right)$ [resp., $\left.\operatorname{wcl}\left(T_{n}(M)\right)\right]$ is complete for each $n \in \mathbb{N}$. By Theorem 12 , for each $n \geq 1$, there exists $\left\{x_{n}\right\}$ in $M$ such that $x_{n}=f\left(x_{n}\right)=g\left(x_{n}\right)=T_{n}\left(x_{n}\right)$.

Again the compactness of $\operatorname{cl}(T(M))$ implies that there exists a subsequence $\left\{T x_{m}\right\}$ of $\left\{T x_{n}\right\}$ such that $T x_{m} \rightarrow z \in$ $\mathrm{cl}(T(M))$. Since $\left\{T x_{m}\right\}$ is a sequence in $T(F(f) \cap F(g))$ and $\operatorname{cl}(T(F(f) \cap F(g))) \subseteq F(f) \cap F(g)$, therefore $z \in F(f) \cap F(g)$. Moreover

$$
x_{m}=T_{m}\left(x_{m}\right)=\left(1-k_{m}\right) q+k_{m} T x_{m} \longrightarrow z .
$$

As $T$ is continuous on $M$, we have $T z=z$. Thus $M \cap F(T) \cap$ $F(f) \cap F(g) \neq \phi$.

Next, weak compactness of $\mathrm{wcl}(T(M))$ implies that there exists a subsequence $\left\{T x_{m}\right\}$ of $\left\{T x_{n}\right\}$ such that $T x_{m}$ converges weakly to $z \in \operatorname{wcl}(T(M))$. Since $\left\{T x_{m}\right\}$ is a sequence in $T(F(f) \cap F(g))$ and $\operatorname{wcl}(T(F(f) \cap F(g))) \subseteq F(f) \cap F(g)$, therefore $z \in F(f) \cap F(g)$. Also we have $(I-T) x_{m} \rightarrow 0$ as $m \rightarrow \infty$. Further, demiclosedness of $I-T$ at 0 implies $z=T z$. Thus $M \cap F(T) \cap F(f) \cap F(g) \neq \phi$.

Corollary 17. Let $M$ be a nonempty subset of a normed [resp., Banach] space $X$ and $T, f$, and $g$ are self-maps of $M$. Suppose that $F(f) \cap F(g)$ is q-star shaped and closed [resp., weakly closed] and $(T, f)$ and $(T, g)$ are Banach operator pairs and satisfy (46). Then $M \cap F(T) \cap F(f) \cap F(g) \neq \phi$ provided that $c l(T(M))$ is compact [resp., wcl $(T(M))$ is weakly compact] and Tis continuous [resp., I - T is demiclosed at 0 , where I stands for identity map].

In Theorem 16, if $\varphi(t)=t$ and $\phi(t)=(1 / k-1) t$ for a constant $k$ with $0<k<1$, then we easily obtain the following result.

Corollary 18. Let $M$ be a nonempty subset of a normed [resp., Banach] space $X$ and $T, f$, and $g$ are self-maps of $M$. Suppose that $F(f) \cap F(g)$ is q-star shaped, $c l(T(F(f) \cap F(g))) \subseteq F(f) \cap$ $F(g)$ [resp., $w c l(T(F(f) \cap F(g))) \subseteq F(f) \cap F(g)]$, and $T, f$, and $g$ satisfy

$$
\|T x-T y\| \leq m_{1}(x, y)+\operatorname{Ln}_{1}(x, y) \quad \forall x, y \in M
$$

where

$$
\begin{aligned}
& m_{1}(x, y) \\
&=\max \{\|f x-g y\|, \operatorname{dist}(f x,[q, T x]), \operatorname{dist}(g y,[q, T y]), \\
&\left.\frac{1}{2}[\operatorname{dist}(g y,[q, T x])+\operatorname{dist}(f x,[q, T y])]\right\}, \\
& n_{1}(x, y)= \min \{[\operatorname{dist}(f x,[q, T x])+\operatorname{dist}(g y,[q, T y])], \\
&\operatorname{dist}(g y,[q, T x]), \operatorname{dist}(f x,[q, T y])\} .
\end{aligned}
$$

Then $M \cap F(T) \cap F(f) \cap F(g) \neq \phi$ provided that $c l(T(M))$ is compact [resp., $\operatorname{wcl}(T(M))$ is weakly compact] and $T$ is continuous [resp., $I-T$ is demiclosed at 0 , where I stands for identity map].

Remark 19. (1) By comparing Theorem 2.3(i) of Abbas and Ilić [12] with the first case of Corollary 18 (when $g=f$ ), their assumptions " $M$ is $q$-star shaped, $\operatorname{cl}(T(M)) \subseteq f(M)$, and $f$ and $T$ are weakly compatible on $M$ " are replaced with " $F(f)$ is $q$-star shaped and $\mathrm{cl}(T(F(f))) \subseteq F(f)$."

(2) By comparing Theorem 2.3(ii) of Abbas and Ilić [12] with the second case of Corollary 18 (when $g=f$ ), their assumptions " $M$ is $q$-star shaped, $\operatorname{cl}(T(M)) \subseteq f(M), f$ and $T$ are weakly compatible on $M, f$ is weakly continuous, and $f-T$ is demiclosed at 0 " are replaced with " $F(f)$ is $q$-star shaped, $\operatorname{wcl}(T(F(f))) \subseteq F(f)$, and $T$ is weakly continuous."

(3) By comparing Theorem 2.4 of Song [21] with the first case of Corollary 18 (when $L=0$ ), his assumptions " $M$ is $q$-star shaped, $\mathrm{cl}(T(M)) \subseteq f(M)) \cap g(M)$, the pairs $(T, f)$ and $(T, g)$ are $C_{q}$-commuting, and $f$ and $g$ are $q$-affine and continuous on $M$ " are replaced with $F(f) \cap F(g)$ is $q$-star shaped and $\operatorname{cl}(T(F(f) \cap F(g)) \subseteq F(f) \cap F(g)$."

(4) By comparing Theorem 2.2(i) of Hussain and Jungck [20] with the first case of Corollary 18 ( when $L=0$ ), their assumptions " $M$ is complete and $q$-star shaped, $f$ and $g$ are continuous and affine on $M, T(M) \subseteq f(M) \cap$ $g(M), q \in F(f) \cap F(g)$, and $(T, f)$ and $(T, g)$ are $R$-subweakly commuting pair on $M^{\text {" }}$ are replaced with " $F(f) \cap F(g)$ is $q$ star shaped and $\operatorname{cl}(T(F(f) \cap F(g))) \subseteq F(f) \cap F(g)$."

(5) By comparing Theorem 2.2(ii) of Hussain and Jungck [20] with the second case of Corollary 18 ( when $L=0$ ), their assumptions " $M$ is weakly compact and $q$-star shaped, $f$ and $g$ are affine and continuous on $M, T(M) \subseteq f(M) \cap g(M), q \epsilon$ $F(f) \cap F(g),(T, f)$ and $(T, g)$ are $R$-subweakly commuting pair on $M$, and $f-T$ is demiclosed at 0 " are replaced with "wcl $(T(M))$ is weakly compact, $F(f) \cap F(g)$ is q-star shaped, $\operatorname{wcl}(T(F(f) \cap F(g))) \subseteq F(f) \cap F(g)$, and $I-T$ is demiclosed at 0 ."

Corollary 20. Let $X$ be a normed space [resp., Banach] $X$ and $T, f$, and $g$ are self-maps of $X$. If $p \in X$ and $D \subseteq B_{M}(p)$, $D_{0}:=D \cap F(f) \cap F(g)$ is q-star shaped, $c l\left(T\left(D_{0}\right)\right) \subseteq D_{0}[$ resp., $\left.w c l\left(T\left(D_{0}\right)\right) \subseteq D_{0}\right], \operatorname{cl}(T(D))$ is compact [resp., wcl $(T(D))$ is weakly compact], $T$ is continuous on $D$ [resp., $I-T$ is 
demiclosed at 0 , where I stands for identity map], and (46) holds for all $x, y \in D$, then $B_{M}(p) \cap F(T) \cap F(f) \cap F(g) \neq \phi$.

Remark 21. Corollary 20 improves Theorem 2.8 of Hussain and Jungck [20], Theorems 3.1-3.4 of Song [21], and Corollary 2.5 of Al-Thagafi and Shahzad [19]. It is also noted that Corollary 3.13 of Akbar et al. [22] is a special case of Corollary 20.

We denote by $\mathfrak{E}_{0}$ the class of closed convex subsets of $X$ containing 0 . For $M \in \mathfrak{E}_{0}$, we define $M_{p}=\{x \in M:\|x\| \leq$ $2\|p\|\}$. Clearly $B_{M}(p) \subseteq M_{p} \in \mathfrak{E}_{0}$.

Theorem 22. Let $X$ be a normed [resp., Banach] space and $T, f, g: X \rightarrow X$. If $p \in X$ and $M \in \mathfrak{E}_{0}$ such that $T\left(M_{p}\right) \subseteq M$, $c l\left(T\left(M_{p}\right)\right)$ is compact [resp., wcl $\left(T\left(M_{p}\right)\right)$ is weakly compact], and $\|T x-p\| \leq\|x-p\|$ for all $x \in M_{p}$, then $B_{M}(p)$ is nonempty, closed, and convex with $T\left(B_{M}(p)\right) \subseteq B_{M}(p)$. If, in addition, $D$ is a subset of $B_{M}(p), D_{0}:=D \cap F(f) \cap F(g)$ is q-star shaped, $c l\left(T\left(D_{0}\right)\right) \subseteq D_{0}\left[\right.$ resp., $\left.w c l\left(T\left(D_{0}\right)\right) \subseteq D_{0}\right], T$ is continuous on $D$ [resp., $I-T$ is demiclosed at 0 , where I stands for identity map], and (46) holds for all $x, y \in D$, then $B_{M}(p) \cap F(T) \cap$ $F(f) \cap F(g) \neq \phi$.

Proof. We may assume that $p \notin M$. If $y \in M \backslash M_{p}$, then $\|y\|>2\|p\|$ and so

$$
\|y-p\| \geq\|y\|-\|p\|>\|p\| \geq \operatorname{dist}(p, M)
$$

Thus $\operatorname{dist}\left(p, M_{p}\right)=\operatorname{dist}(p, M)$. Assume that $\mathrm{cl}\left(T\left(M_{p}\right)\right)$ is compact; then by the continuity of norm, there exist $z \in$ $\operatorname{cl}\left(T\left(M_{p}\right)\right)$ such that $\|z-p\|=\operatorname{dist}\left(p, \operatorname{cl} T\left(M_{p}\right)\right)$.

If we assume that $\operatorname{wcl}\left(T\left(M_{p}\right)\right)$ is weakly compact, then by using Lemma 5.5 of $[24$, p. 192] we can show the existence of $z \in \operatorname{wcl}\left(T\left(M_{p}\right)\right)$ such that $\|z-p\|=\operatorname{dist}\left(p, \operatorname{wcl} T\left(M_{p}\right)\right)$. Thus, in both cases, we have

$$
\begin{aligned}
\operatorname{dist}\left(p, M_{p}\right) & \leq \operatorname{dist}\left(p, \operatorname{cl} T\left(M_{p}\right)\right) \leq \operatorname{dist}\left(p, T\left(M_{p}\right)\right) \\
& \leq\|T x-p\| \leq\|x-p\|,
\end{aligned}
$$

for all $x \in M_{p}$. It follows that $\|z-p\|=\operatorname{dist}(p, M)$. Thus $B_{M}(p)$ is nonempty, closed, and convex with $T\left(B_{M}(p)\right) \subseteq$ $B_{M}(p)$. The compactness of $\operatorname{cl}\left(T\left(M_{p}\right)\right)$ [resp., weak compactness of $\operatorname{wcl}\left(T\left(M_{p}\right)\right)$ ] implies that $\mathrm{cl}(T(D))$ is compact [resp., $\mathrm{wcl}(T(D))$ is weakly compact]. Then by Corollary $20, B_{M}(p) \cap$ $F(T) \cap F(f) \cap F(g) \neq \phi$.

Remark 23. (i) Theorem 22 extends Theorems 4.1 and 4.2 of Al-Thagafi [17], Theorem 2.6 of Al-Thagafi and Shahzad [19], Theorem 2.14 of Hussain and Jungck [20], and Theorem 8 of Habiniak [23].

(ii) Theorem 3.15 of Akbar et al. [22] is a particular case of Theorem 22.

\section{Examples}

Now, in order to support the usability of our results, we present the following nontrivial examples.
Example 24. Let $X=[0, \infty)$ be endowed with the Euclidean metric $d(x, y)=|x-y|$ and let $M=\{0,1,2,3\}$ and define $T, f, g: M \rightarrow M$ by

$$
\begin{gathered}
T=\left(\begin{array}{llll}
0 & 1 & 2 & 3 \\
0 & 0 & 0 & 2
\end{array}\right), \quad f=\left(\begin{array}{llll}
0 & 1 & 2 & 3 \\
0 & 1 & 3 & 1
\end{array}\right), \\
g=\left(\begin{array}{llll}
0 & 1 & 2 & 3 \\
0 & 3 & 1 & 3
\end{array}\right) .
\end{gathered}
$$

Here $F(f)=\{0,1\}$ and $F(g)=\{0,3\}$ so that $F(f) \cap F(g)=\{0\}$ is nonempty and $\mathrm{cl}(T(F(f) \cap F(g)))=\{0\} \subseteq F(f) \cap F(g)$. Also $\mathrm{cl}(T(M))=\{0,2\}$, being a finite set, is complete. Define $\varphi, \phi:[0, \infty) \rightarrow[0, \infty)$ by

$$
\varphi(t)=t^{2}, \quad \phi(t)=\frac{t}{2} .
$$

Now we will show that $T$ is generalized $(\varphi, \phi, L)_{f, g}$-weak contraction for $L \geq 7 / 2$. Let $x, y \in M$ with $x \neq y$ and consider the following possible cases.

(i) If $(x, y) \in\{(0,3),(3,1),(2,3)\}$, then $d(T x, T y)=2$, $m(x, y)=3$, and $n(x, y)=1$. So,

$$
\begin{gathered}
\varphi(m(x, y))-\phi(m(x, y))+L \varphi(n(x, y)) \\
=\varphi(3)-\phi(3)+L \varphi(1) \\
=\frac{15}{2}+L>4=\varphi(d(T x, T y)),
\end{gathered}
$$

for all $L \geq 0$.

(ii) If $x=3, y=0$, then $d(T x, T y)=2, m(x, y)=3 / 2$, and $n(x, y)=1$. So,

$$
\begin{gathered}
\varphi(m(x, y))-\phi(m(x, y))+L \varphi(n(x, y)) \\
=\varphi\left(\frac{3}{2}\right)-\phi\left(\frac{3}{2}\right)+L \varphi(1) \\
=\frac{3}{2}+L \geq 4=\varphi(d(T x, T y)),
\end{gathered}
$$

for all $L \geq 5 / 2$.

(iii) If $x=1, y=3$, then $d(T x, T y)=2, m(x, y)=2$, and $n(x, y)=1$. So,

$$
\begin{gathered}
\varphi(m(x, y))-\phi(m(x, y))+L \varphi(n(x, y)) \\
=\varphi(2)-\phi(2)+L \varphi(1) \\
=3+L \geq 4=\varphi(d(T x, T y)),
\end{gathered}
$$

for all $L \geq 1$.

(iv) If $x=3, y=2$, then $d(T x, T y)=2, m(x, y)=1$, and $n(x, y)=1$. So,

$$
\begin{gathered}
\varphi(m(x, y))-\phi(m(x, y))+L \varphi(n(x, y)) \\
=\varphi(1)-\phi(1)+L \varphi(1) \\
=\frac{1}{2}+L \geq 4=\varphi(d(T x, T y)),
\end{gathered}
$$

for all $L \geq 7 / 2$. 
(v) If $(x, y) \in\{(0,1),(1,0),(0,2),(2,0),(1,2),(2,1)\}$, then $d(T x, T y)=0$ and hence inequality (5) is obviously satisfied.

So, in view of the above discussion, we concluded that $T$ is generalized $(\varphi, \phi, L)_{f, g}$-weak contraction for $L \geq 7 / 2$. Therefore, all the conditions of Theorem 12 are satisfied and hence $T, f$, and $g$ have a common fixed point. Here it is seen that 0 is the unique common fixed point of $T, f$, and $g$.

Remark 25. (1) In Example 24, it is to be noted that the maps $T, f$, and $g$ do not satisfy the conditions (1.1) and (3.1) of [22] at the points $x=3, y=0 ; x=1, y=3$ and $x=3, y=$ 2. Therefore, Theorems 2.2 and 3.3 of [22] cannot apply to Example 24.

(2) In Example 24, the maps $T, f$, and $g$ do not satisfy the condition (3.1) of [26] at the points $x=3, y=0 ; x=0, y=3$. Therefore, Theorem 3.5 of [26] cannot apply to Example 24.

Example 26. Let $X=M=[0,1 / 2) \cup(1 / 2,1]$ be endowed with the Euclidean metric $d(x, y)=|x-y|$ and define $T: M \rightarrow M$ by

$$
T(x)= \begin{cases}\frac{1}{3}, & x \in\left[0, \frac{1}{2}\right) \cup\left(\frac{1}{2}, \frac{2}{3}\right), \\ \frac{2}{5}, & x \in\left[\frac{2}{3}, 1\right] .\end{cases}
$$

We observe that $\mathrm{cl}(T(M))=\{1 / 3,2 / 5\} \subseteq M$ and also being a finite set $\operatorname{cl}(T(M))$ is complete. Define the function $\varphi, \phi$ : $[0, \infty) \rightarrow[0, \infty)$ by

$$
\varphi(t)=2 t, \quad \phi(t)=\frac{3}{2} t, \quad \forall t \geq 0 .
$$

Now, we prove that $T$ is generalized $(\varphi, \phi, L)$-weak contraction mapping for $L \geq 0$, that is, to verify the following inequality:

$$
\begin{aligned}
& \varphi(d(T x, T y)) \\
& \quad \leq \varphi(M(x, y))-\phi(M(x, y))+L \varphi(N(x, y)),
\end{aligned}
$$

where

$$
\begin{gathered}
M(x, y)=\max \{d(x, y), d(T x, x), d(T y, y), \\
\left.\frac{1}{2}[d(y, T x)+d(x, T y)]\right\}, \\
N(x, y)=\min \{[d(T x, x)+d(T y, y)], \\
d(y, T x), d(x, T y)\} .
\end{gathered}
$$

If $x=y \in M$, then $d(T x, T y)=0$ and hence inequality (62) trivially holds. Let $x, y \in M$ with $x \neq y$; then we have the following two possibilities.

(i) If $x \in[0,1 / 2) \cup(1 / 2,2 / 3), y \in[2 / 3,1]$, then $d(T x, T y)=1 / 15$ and $d(y, T y) \in[4 / 15,3 / 5]$.
Therefore

$$
\begin{aligned}
\varphi(d(T x, T y)) & =\frac{2}{15} \leq \frac{1}{2} d(y, T y) \leq \frac{1}{2} M(x, y) \\
& =2 M(x, y)-\frac{3}{2} M(x, y) \\
& =\varphi(M(x, y))-\phi(M(x, y)) .
\end{aligned}
$$

This yields that

$$
\begin{aligned}
& \varphi(d(T x, T y)) \\
& \quad \leq \varphi(M(x, y))-\phi(M(x, y))+L \varphi(N(x, y))
\end{aligned}
$$

holds for all $L \geq 0$

(ii) If $x \in[2 / 3,1], y \in[0,1 / 2) \cup(1 / 2,2 / 3)$, then by interchanging the role of $x$ and $y$ in case (i), inequality (62) follows.

Thus we checked that $T$ is generalized $(\varphi, \phi, L)$-weak contraction for all $L \geq 0$. Therefore, all the conditions of Corollary 8 are satisfied and hence $T$ has a fixed point in $M$. Here it is seen that $1 / 3$ is the unique fixed point of $T$.

Remark 27. In Example 26, $X$ is not complete since the sequence $\{(1 / 2)(1-1 / n)\}$ is not Cauchy in $X$. Therefore, Theorem 2.2 of [5], Theorem 2.1 of [6], Theorem 2.4 of [11], and Corollary 3.1 of [25] cannot apply to Example 26.

Remark 28. Theorem 12 and first case of Theorem 7 are not true if we relax the condition $\mathrm{cl}(T(F(f) \cap F(g))) \subseteq F(f) \cap F(g)$ and $\mathrm{cl}(T(M)) \subseteq f(M) \cap g(M)$, respectively. This can be seen by the following example.

Example 29. Let $X=R$ with the usual metric and $M=[0,1]$. Define $T, f, g: M \rightarrow M$ by

$$
\begin{aligned}
& T(x)= \begin{cases}\frac{1}{3}, & x \in\left[0, \frac{1}{2}\right) \cup\left(\frac{1}{2}, \frac{2}{3}\right), \\
0, & x \in\left[\frac{2}{3}, 1\right], \\
1, & x=\frac{1}{2},\end{cases} \\
& f(x)= \begin{cases}\frac{1}{2}, & x \in\left[0, \frac{2}{3}\right)-\left\{\frac{1}{3}\right\}, \\
0, & x \in\left[\frac{2}{3}, 1\right], \\
\frac{1}{3}, & x=\frac{1}{3},\end{cases} \\
& g(x)= \begin{cases}\frac{1}{2}, & x \in\left[0, \frac{1}{2}\right] \cup\left[\frac{2}{3}, 1\right], \\
x, & x \in\left(\frac{1}{2}, \frac{2}{3}\right) .\end{cases}
\end{aligned}
$$

Then $F(f)=\{1 / 2,1 / 3\}, F(g)=[1 / 2,2 / 3)$; therefore $F(f) \cap$ $F(g)=\{1 / 2\}$ is nonempty. Also, $\operatorname{cl}(T(M))=\{0,1 / 3,1\}$, being 
a closed subset of complete metric space, is complete. Further, $f(M)=\{0,1 / 3,1 / 2\}$ and $g(M)=[1 / 2,2 / 3)$ gives $f(M) \cap$ $g(M)=\{1 / 2\}$. Here, we observe that $\mathrm{cl}(T(F(f) \cap F(g)))=$ $\{1\} \nsubseteq F(f) \cap F(g)$ and $\mathrm{cl}(T(M)) \nsubseteq f(M) \cap g(M)$.

Define the function $\varphi, \phi:[0, \infty) \rightarrow[0, \infty)$ by

$$
\varphi(t)=t, \quad \phi(t)=t^{2}, \quad \forall t \geq 0
$$

Then by using the routine calculation as is done in Example 24 , one can easily check that $T$ is generalized $(\varphi, \phi, L)_{f, g^{-}}$ weak contraction for all $L \geq 6$. Hence all the conditions of Theorem 12 and first case of Theorem 7 except $\mathrm{cl}(T(F(f) \cap$ $F(g))) \subseteq F(f) \cap F(g)$ and $\operatorname{cl}(T(M)) \subseteq f(M) \cap g(M)$, respectively, are satisfied. Note that $F(T) \cap F(f) \cap F(g)=\phi$, $C(f, T)=\{1 / 3\} \cup[2 / 3,1]$, and $C(g, T)=\phi$.

Remark 30. (1) In Example 24, $T$ is generalized $(\varphi, \phi, L)_{f, g^{-}}$ weak contraction for $L \geq 7 / 2$ but it is not generalized $(\varphi, \phi)_{f, g}$-weak contraction at $x=3, y=0$ for any $\varphi$ and $\phi$ defined in (5) and hence $T$ is not generalized $\phi_{f, g}$-weak contraction. Note that $F(T) \cap F(f) \cap F(g)=\{0\}$.

(2) In Example 29, $T$ is generalized $(\varphi, \phi, L)_{f, g}$-weak contraction for $L \geq 6$ but it is not generalized $(\varphi, \phi)_{f, g}$-weak contraction at $x=2 / 3, y=1 / 2$ for any $\varphi$ and $\phi$ defined in (5) and hence $T$ is not generalized $\phi_{f, g}$-weak contraction. Note that $F(T) \cap F(f) \cap F(g)=\phi$.

\section{Conclusions}

We introduce a new class of generalized $(\varphi, \phi, L)_{f, g}$-weak contraction as a generalization of several known contractions and present a sufficient condition for the existence and uniqueness of common fixed point. Moreover, we also prove some invariant approximation result as an application and supporting examples are also given.

\section{Conflict of Interests}

The authors declare that they have no conflict of interests regarding the publication of this paper.

\section{Acknowledgment}

The authors would like to thank the anonymous reviewers for their valuable comment which helps to improve the contents and presentation of this paper.

\section{References}

[1] Y. I. Alber and S. Guerre-Delabriere, "Principle of weakly contractive maps in Hilbert spaces," in New Results in Operator Theory and Its Applications, Y. Lyubich, Ed., vol. 98 of Operator Theory: Advances and Applications, pp. 7-22, Birkhäuser, Basel, Switzerland, 1997.

[2] B. E. Rhoades, "Some theorems on weakly contractive maps," Nonlinear Analysis-theory Methods \& Applications, vol. 47, no. 4, pp. 2683-2693, 2001.
[3] Q. Zhang and Y. Song, "Fixed point theory for generalized $\varphi$ weak contractions," Applied Mathematics Letters, vol. 22, no. 1, pp. 75-78, 2009.

[4] M. S. Khan, M. Swaleh, and S. Sessa, "Fixed point theorems by altering distances between the points," Bulletin of the Australian Mathematical Society, vol. 30, no. 1, pp. 1-9, 1984.

[5] D. Doric, "Common fixed point for generalized $(\psi, \varphi)$-weak contractions," Applied Mathematics Letters, vol. 22, no. 12, pp. 1896-1900, 2009.

[6] M. Abbas and D. Đorić, "Common fixed point theorem for four mappings satisfying generalized weak contractive condition," Filomat, vol. 24, no. 2, pp. 1-10, 2010.

[7] A. Razani, V. Parvaneh, and M. Abbas, "A common fixed point for generalized $(\psi, \phi)_{f, g}$-weak contractions," Ukrainian Mathematical Journal, vol. 63, no. 11, pp. 1756-1769, 2012.

[8] V. Berinde, "Approximating fixed points of weak contractions using the Picard iteration," Nonlinear Analysis Forum, vol. 9, no. 1, pp. 43-53, 2004.

[9] G. V. R. Babu, M. L. Sandhya, and M. V. R. Kameswari, "A note on a fixed point theorem of Berinde on weak contractions," Carpathian Journal of Mathematics, vol. 24, no. 1, pp. 8-12, 2008.

[10] V. Berinde, "General constructive fixed point theorems for Ćirić-type almost contractions in metric spaces," Carpathian Journal of Mathematics, vol. 24, no. 2, pp. 10-19, 2008.

[11] V. Berinde, "Some remarks on a fixed point theorem for Ćirićtype almost contractions," Carpathian Journal of Mathematics, vol. 25, no. 2, pp. 157-162, 2009.

[12] M. Abbas and D. llić, "Common fixed points of generalized almost nonexpansive mappings," Filomat, vol. 24, no. 3, pp. 11$18,2010$.

[13] G. Jungck, "Compatible mappings and common fixed points," International Journal of Mathematics and Mathematical Sciences, vol. 9, no. 4, pp. 771-779, 1986.

[14] G. Jungck, "Common fixed points for noncontinuous nonself maps on nonmetric spaces," Far East Journal of Mathematical Sciences, vol. 4, no. 2, pp. 199-215, 1996.

[15] N. Shahzad, "Invariant approximations and $R$-subweakly commuting maps," Journal of Mathematical Analysis and Applications, vol. 257, no. 1, pp. 39-45, 2001.

[16] J. Chen and Z. Li, "Common fixed-points for Banach operator pairs in best approximation," Journal of Mathematical Analysis and Applications, vol. 336, no. 2, pp. 1466-1475, 2007.

[17] M. A. Al-Thagafi, "Common fixed points and best approximation," Journal of Approximation Theory, vol. 85, no. 3, pp. 318323, 1996.

[18] M. A. Al-Thagafi and N. Shahzad, "Noncommuting selfmaps and invariant approximations," Nonlinear Analysis: Theory, Methods \& Applications, vol. 64, no. 12, pp. 2778-2786, 2006.

[19] M. A. Al-Thagafi and N. Shahzad, "Banach operator pairs, common fixed-points, invariant approximations, and *nonexpansive multimaps," Nonlinear Analysis: Theory, Methods \& Applications, vol. 69, no. 8, pp. 2733-2739, 2008.

[20] N. Hussain and G. Jungck, "Common fixed point and invariant approximation results for noncommuting generalized $(f, g)$ nonexpansive maps," Journal of Mathematical Analysis and Applications, vol. 321, no. 2, pp. 851-861, 2006.

[21] Y. Song, "Common fixed points and invariant approximations for generalized $(f, g)$-nonexpansive mappings," Communications in Mathematical Analysis, vol. 2, no. 2, pp. 17-26, 2007. 
[22] F. Akbar, A. R. Khan, and N. Sultana, "Common fixed point and approximation results for generalized $(f, g)$-weak contractions," Fixed Point Theory and Applications, vol. 2012, article 75, 2012.

[23] L. Habiniak, "Fixed point theorems and invariant approximations," Journal of Approximation Theory, vol. 56, no. 3, pp. 241$244,1989$.

[24] S. Singh, B. Watson, and P. Srivastava, Fixed Point Theory and Best Approximation: The KKM-Map Principle, vol. 424 of Mathematics and its Applications, Kluwer Academic, Dordrecht, The Netherlands, 1997.

[25] M. Jleli, E. Karapınar, and B. Samet, "Fixed point results for almost generalized cyclic $(\psi, \varphi)$-weak contractive type mappings with applications," Abstract and Applied Analysis, vol. 2012, Article ID 917831, 17 pages, 2012.

[26] S. Rathee and A. Kumar, "Some common fixed-point and invariant approximation results with generalized almost contractions," Fixed Point Theory and Applications, vol. 2014, article 23, 2014.

[27] A. Kumar, S. Rathee, and N. Kumar, "The point of coincidence and common fixed point for three mappings in cone metric spaces," Journal of Applied Mathematics, vol. 2013, Article ID 146794, 6 pages, 2013.

[28] N. Hussain and Y. J. Cho, "Weak contractions, common fixed points, and invariant approximations," Journal of Inequalities and Applications, vol. 2009, Article ID 390634, 10 pages, 2009. 


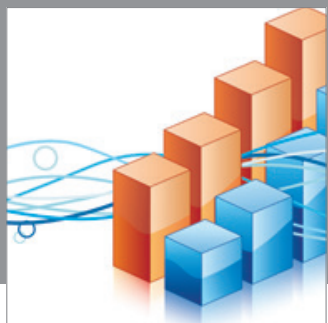

Advances in

Operations Research

mansans

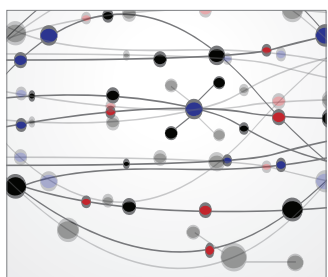

The Scientific World Journal
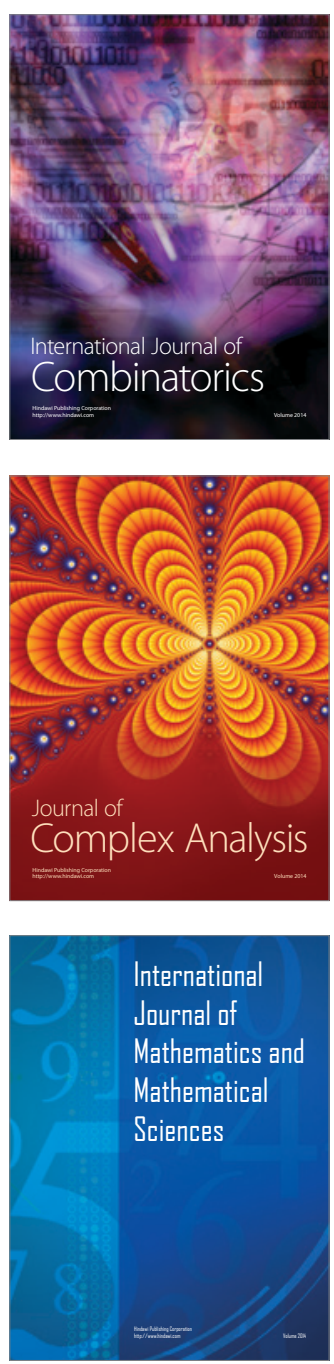
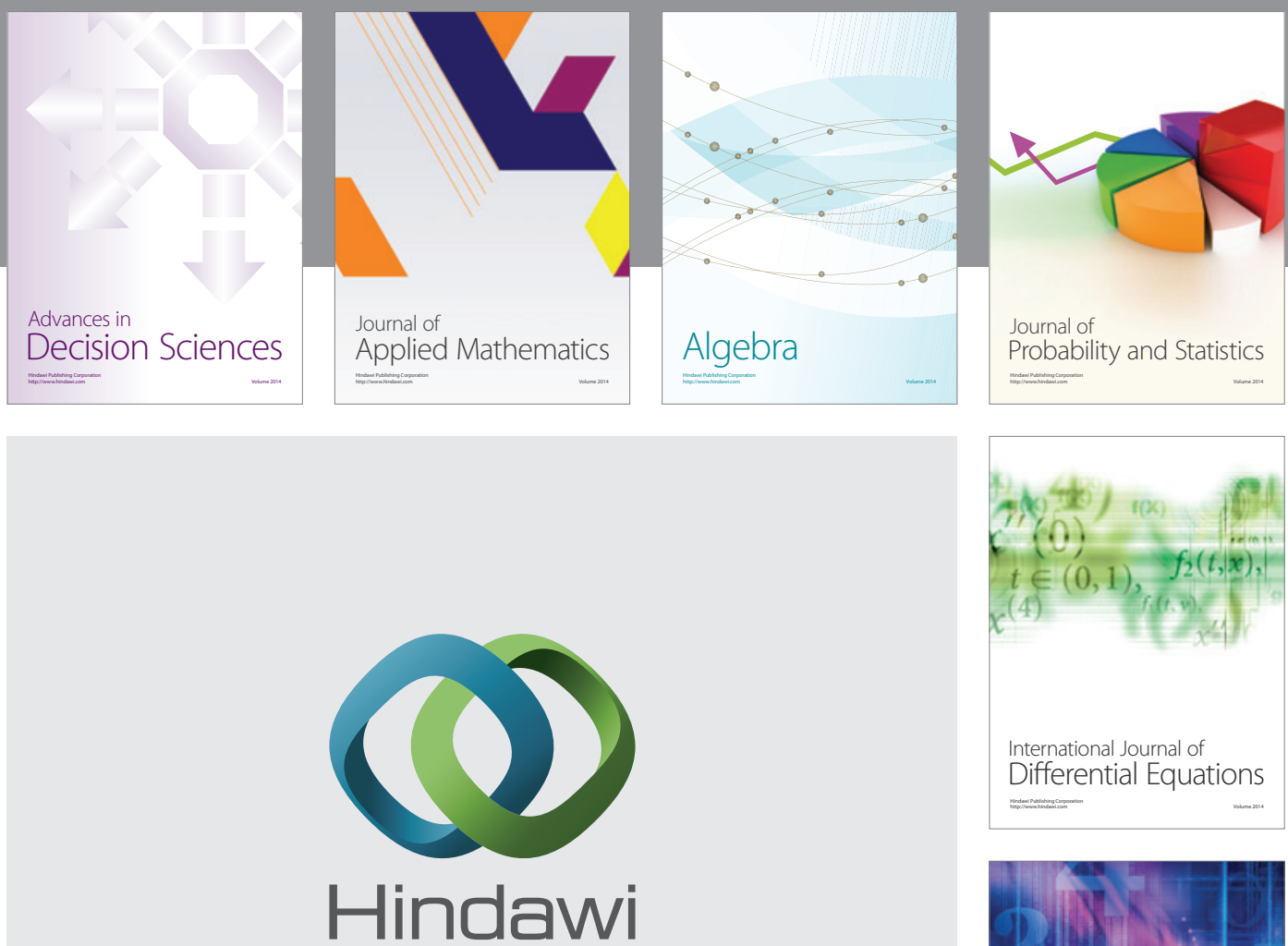

Submit your manuscripts at http://www.hindawi.com
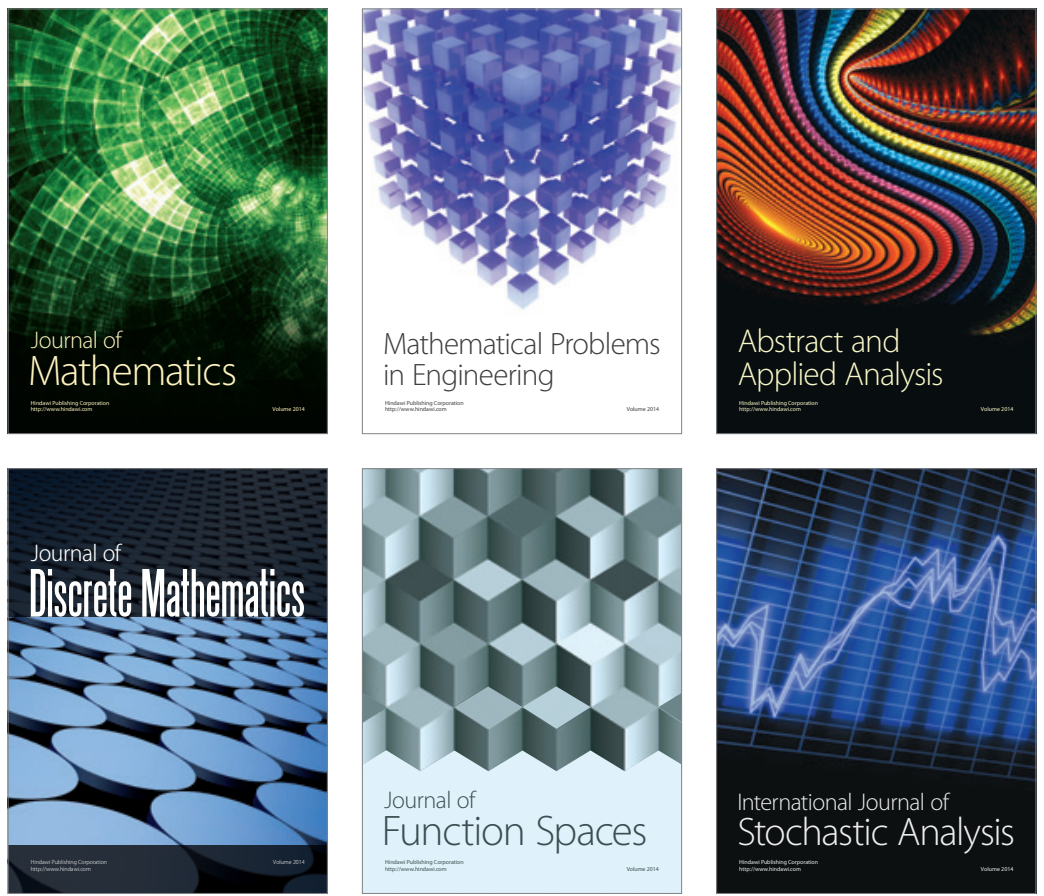

Journal of

Function Spaces

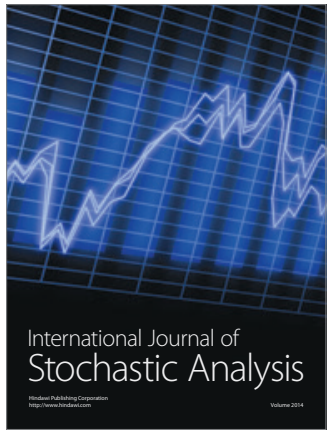

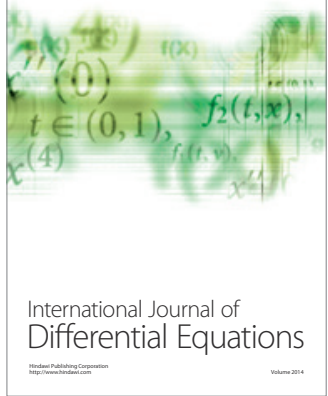
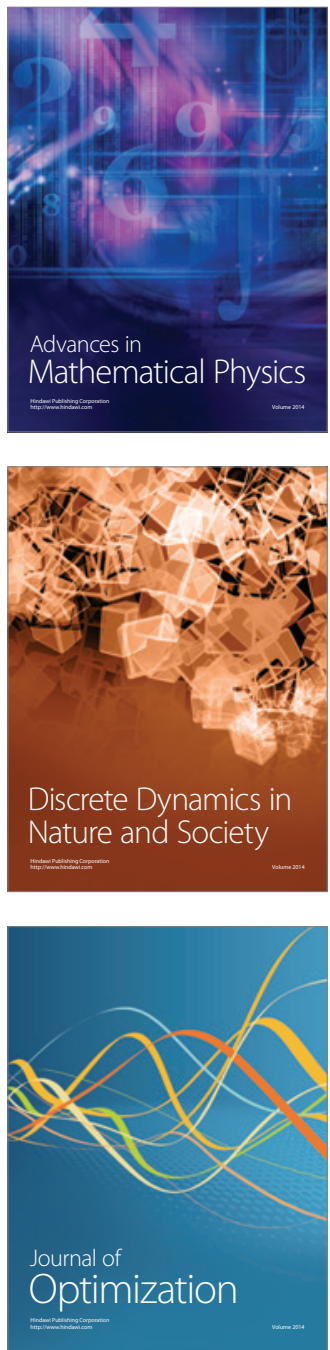